\title{
Polypeptide growth factors: Structure, function and mechanism of action
}

\author{
Ralph A. Bradshaw, Ritsuko Fujii, Hubert Hondermarck, Simona Raffioni, \\ Yvonne Wu and Michael A. Yarski \\ Department of Biological Chemistry, College of Medicine, University of California, Irvine, CA 92717, USA.
}

\begin{abstract}
Polypeptide growth factors are a diverse group of hormone-like agents that regulate growth and differentiation through cell surface receptors. They are generally represented by homologous families containing several members with distinct overlapping receptor interactions and hence, responsive tissue specificities. Similarly, their receptors are also clustered in family groups of sequence-related proteins. The neurotrophin group, characterized by its typical prototypical member nerve growth factor (NGF), has four members which interact variably with three receptors of the trk family. The activation of their tyrosine kinases initiates the characteristic responses. In PC12 cells, stimulation by NGF leads to activation of non-receptor tyrosine kinases and several phospholipid dependent pathways. The sum of these signals induce a variety of immediate early response genes that govern the phenotypic response. However, the minimum pathway (and its essential components) is not yet fully defined.
\end{abstract}

One of the major functions of proteins is to mediate signal transduction. In higher organisms, this complex process utilizes both soluble and membrane bound ligands to initiate the responses and a wide variety of effector molecules, including cell-surface receptors, that are generally ligand specific, and the component of the intracellular machinery that translate the stimulus into the characteristic phenotypic response (ref. 1). Of the many biological responses that are controlled in this fashion, those related to growth are now amongst those that are best studied. In general, these can be subdivided into hyperplastic (increase in cell number) and hypertrophic (increase in cell size), and are, for the most part, controlled by polypeptide growth factors. These substances are basically indistinguishable in their principal functional and mechanistic properties from many of the classic hormones (ref. 2). In the main, they act through specific cell surface receptors that can initiate a variety of intracellular signals invariably involving a spectrum of protein phosphorylations (ref. 3 ).

As with the classical hormone, the polypeptide growth factors are usually relatively small, highly soluble proteins of compact structure. Although some are glycosylated, the majority are not (ref. 4). Most also contain several disulfide bonds which lends further reinforcement to the 3-dimensional structure, and is a feature expected of proteins exported through the endoplasmic reticulum (ref. 5). There is, however, a small but significant subgroup that is devoid of signal peptides and possess only reduced half-cysteine residues, as expected for intracellular proteins. Significant quantities of these agents are often found associated with the extracellular matrix, but the manner in which they reach extracellular compartment is not presently understood.

From a functional point of view, polypeptide growth factors can conveniently be divided into four categories (Table 1). The best known group are those which act on tissues and include a broad range of agents with a myriad of responsive target cells. The neurotrophic factors are a more specialized

Table 1. Functional classes of polypeptide growth factors.

Tisse growth factors e.g. Epidermal growth factors

Hemopoietic growth factors e.g. Erythropoietin

Neurotrophic factors e.g. Nerve growth factor

Cytokines e.g. Interleukin-1 
grouping and are now generally considered to be those entities which act on neurons. In this capacity, these factors can only act as trophic agents (because their target cells are post-mitotic). The hemopoietic growth factors and cytokines act on the circulating cells and those involved in the immune response (ref. 4). It is important to realize that there is considerable overlap in the activities of many of these factors and many can be listed in more than one category. It is also important to note that the various cells and tissues of origin of the different classes of polypeptide growth factors are equally diverse.There are in fact some factors that have been shown to influence such a wide variety of cells that they can be assigned to all four categories.

Like most proteins, polypeptide growth factors can also be subdivided into "families". This is well illustrated by the insulin related growth factors (IGFs), the fibroblast growth factor (FGFs) and, more recently, the neurotrophins (NTs) (Table 2). These relationships are defined by extensive identities in the amino acid sequences as well as other markers of homologous relationships. In addition, the various receptors for these families show a similar relationship. Thus, each family of ligands has a corresponding family of receptors and crossover in both binding and function (albeit, often with different affinities) is commonly but not universally observed. Thus, there is potential for a large number of possible ligand receptor combinations that can be controlled simply by expression of either entity in different cells, tissues and organs. From a physiological perspective, this greatly increases the regulatory opportunities for the target tissues without greatly increasing the number of known factors (and their receptors).

\section{NGF AND THE NEUROTROPHINS}

\section{Structural aspects}

Neurotrophic substances are generally defined as those exhibiting positive influences on neurons of either the peripheral or central nervous systems (ref. 6). These responses include neurite proliferation, maintenance of viability, and induction of neural-specific genes. Table 3 provides a partial list of substances which meet these criteria.

Nerve growth factor (NGF) is clearly the best known of the neurotrophic factors and is considered prototypical for substances which function as chronic stabilizers of specific neuronal populations. It was the first growth factor to be identified (ref.7) and remains the most extensively studied of the neurotrophic factor subgroup (ref. 8).

The chemical properties of nerve growth factors are based primarily on studies with the protein obtained from mouse submandibular glands. The first amino acid sequence was determined from protein from this source (ref.9), although amino acid sequences from several sources are now known (ref. 10). Many of these have been determined from cDNA or genomic sequences which have, in turn, revealed the organization of the precursor which must be activated by limited proteolysis to produce the mature protein. This latter structure, which occurs as a homodimer of protomers containing 118 amino acids (ref. 11), has been recently determined by X-ray crystallographic means (ref. 12). These studies have revealed that the NGF subunit has an elongated fold composed of four principal strands of $\beta$-pleated sheet anchored at one end by the three intrachain disulfide bonds which occur in a tight cluster. One end of the molecule is characterized by three loops, which are further stabilized by shorter segments of $\beta$-sheet, while the opposite end contains a single loop without defined secondary structure. Neither the amino nor carboxyl-terminal sequences were visible in the crystal employed. The dimeric structure is organized so that the long axes of each protomer are roughly parallel with most of the dimer interface contributed by residues found in the four long structures of $\beta$-sheet. Not unexpectedly, the four major loops contain the majority of the residues that are variant amongst the many NGF sequences and, perhaps more importantly, are also the sites of differences between the members of the neurotrophin family. These latter differences suggest that these loop areas define the receptor interactions that distinguish the neurotrophin family. Residues contributing to the binding site of low affinity receptor (see below) have been partially defined by site directed mutagenesis and occur in 2 of these loop regions (ref. 13). 
Table 2. Some polypeptide growth factor receptor families and their ligands.

\begin{tabular}{ll}
\hline Receptors & Ligand \\
\hline FGFs & \\
FGFR-1 (flg) & bFGF, aFGF, FGF-4 \\
FGFR-2 (bek) & bFGF, aFGF, FGF-4 \\
FGFR-2 (ksam) & KGF \\
FGFR-3 (cek2) & bFGF, aFGF \\
FGFR-4 & aFGF, FGF-4, FGF-6 \\
IGFs & \\
INSULIN R & INSULIN, IGF-I \\
IGF-IR & INSULIN. IGF-I, IGF-II \\
IGF-IIR & IGF-I, IGF-II \\
Neurotrophins & \\
p140trkA & NGF, NT3, NT4/5 \\
p145trkB & BDNF, NF4/5 \\
p145trkC & NT3, NT4/5 \\
p75 & NGF, BDNF, NT3, NT4/5 \\
\hline
\end{tabular}

Key: FGF: fibroblast growth factor KGF: keratinocyte growth factor IGF: insulin like growth factor NT: neurotrophin BDNF: brain derived neurotrophic factor
Table 3. Some neurotrophic factors

Neurotrophins
Nerve growth factor
Brain-derived neurotrophic factor
Neurotrophin-3
Neurotrophin- $4 / 5$
Ciliary neurotrophic factor
FGF
EGF
IGF-I \& II
Glial- derived nexin
Purpurin
Platelet-derived growth factor

Table 4. Growth factor induced signaling

Processes
Tyrosine phosphorylation
Serine/Threonine phosphorylation
Phospholipid hydrolysis
Ion transport
Receptor ligand internalization
Gene transcription
Components
Receptor (tyrosine kinase)
Phospholipase C (and A2)
Protein kinase C
Diacylglycerol and phosphoinositols
src (src-like proteins)
ras
Ion transporters
Other kinases (MAP kinase, etc.)

Recently, based on purely structural considerations, it has been possible to associate NGF with a superfamily composed of two additional extensive growth factor families (ref. 14, 15, R.A. Bradshaw, J. Murray-Rust, and T.L. Blundell, unpublished observations). The structures of human platelet-derived growth factor (BB PDGF) and human transforming growth factor $\beta-2$ (TGF $\beta$ ) have also been determined by X-ray crystallographic methods (ref. 16, 17,18) and each has been found to contain a core structure of $\beta$-pleated sheet that are similar in orientation to that found in NGF. In addition, each contains the same cluster of disulfide bonds with a superimposible bonding pattern. Interestingly, the loop structures are quite variant, both in size and orientation. Furthermore, although PDGF and TGF both occur as dimeric structures, they are held together, in part, by intrachain disulfide bonds and the orientation of the subunits within each dimer is head-to-tail (as opposed to the head-to-head relationship found in NGF). Clearly, substantive change has been introduced into these molecules as a result of evolutionary events that has allowed each family to develop its own unique characteristics. This is also reflected in the fact that there is little similarity evident at the receptor level. Both NGF and PDGF utilize receptors containing tyrosine kinases, whereas the TGF family apparently does not (ref. 19,20). There is no apparent similarity in the ligand binding portions of the NGF and PDGF receptor families. Nonetheless, it is probably significant that all three members of this superfamily have retained their dimeric subunit structures and that the same loops have been indicated to be involved in receptor interactions in at least two (NGF and PDGF) of the three subgroups. It may also prove to be correct that these same loops are involved in TGF receptor interactions as well.

The neurotrophin family is presently considered to be composed of five members: NGF, brain derived neurotrophic factor (BDNF), neurotrophin 3 (NT3), neurotrophin 4 (NT4), and neurotrophin 5 (NT5)(ref. 21). NT4 and NT5 may represent only species variations rather than true separate members of the family. All members of the families contain the same basic structure as found in NGF, including extensive sequence similarities (approximately $50 \%$ ) complete conservation 
of the disulfide structure and, in all probability, the same 3-dimensional structure. As indicated in Table 2, the various neurotrophins interact differentially with different members of the NGF receptor (trk) family (see below). Variations in expression and tissue distribution suggest that different neurotrophins have different functional roles in both the development and maintenance of neurons in the peripheral and central nervous systems (ref. 10).

\section{Functional aspects}

Receptors. Two different receptors for nerve growth factor have been identified (ref. 22). The first of these, designated LNGFR or p75, is widely distributed on both neuronal and non-neuronal cells. The latter are not responsive. This cell surface glycoprotein binds NGF with low affinity $\left(10^{-9} \mathrm{M}\right)$ and has a measured molecular weight of approximately $75 \mathrm{kDa}$ (although the true molecular weight is probably somewhat lower). Amino acid sequence analysis, determined from the corresponding cDNAs (ref. 23,24) reveals the usual 3-domain structure for cell-surface receptors (extracellular, transmembrane, intracellular) but an absence of a known effector sequence in the last region). The second receptor, now termed $\mathrm{p}^{140 \mathrm{c}-t r k}$, is the product of the trkA proto-oncogene that was identified from a corresponding transforming version isolated from colon carcinoma cells. It binds NGF with high affinity $\left(10^{-11} \mathrm{M}\right)$, although this remains somewhat controversial (ref. 22$)$. The sequence of this protein, also determined from cDNA, indicated the presence of a tyrosine kinase domain (as well as transmembrane and extracellular domain in the usual orientation). It now seems clear that this protein, is the principal signal generator for NGF, and that the homologous proteins $p^{145 c-t r k} \mathrm{~B}$ and $\mathrm{p}$ ${ }^{145 \mathrm{c}-t r k} \mathrm{C}$, play a similar role for the other neurotrophins (see table 2). It should be pointed out, however, that the relationship between $\mathrm{p} 75$ and $\mathrm{p}^{140 \mathrm{c}-\mathrm{rk} k} \mathrm{~A}$ has not been completely resolved and it remains to be determined whether p75 may play some functional role in NGF-induced signal transduction (ref. 10,22,25).

Signal transduction. Although the activation of the receptor bound tyrosine kinases clearly is the principal initiating event for NGF, PDGF, FGF and the other growth factors of this type, there are a considerable number of additional downstream elements that are essential to manifest the full biological response in any target cell. Table 4 lists several of the processes and associated components that have been identified in the NGF response (both in primary and cultured cells). These pathways are assembled as cascades in which the tyrosine phosphorylations induced by the receptor kinase (following its own autophosphorylations and subsequent association of potential substrates through SH2 domains) lead to activation and subsequent generation of activity. This is illustrated in Fig. 1, which shows two sets of potential cascade pathways. The first, which results from the activation of phospholipase $c \gamma(\mathrm{PLC} \gamma)$ leads to the cleavage of phosphoinositides and the production of corresponding inositol phosphates and diacyl glycerides. Each, in turn, can act as an additional signal transducer with the former causing the release of intracellular calcium and with the latter directly activating protein kinase $\mathrm{C}(\mathrm{PKC})$, that is induced to associate with the membrane. Similarly, G-protein activating protein (GAP) can be phosphorylated resulting in its activation and the consequent stimulation of Ras protein, a constituent known to be involved in NGF activity (ref. $26,27)$. There are clearly additional pathways (represented by the central arrow in Fig. 1) that may also be involved. These include the activation of phosphoinositol-3-kinase and raf kinase (ref. $28,29,30)$.

Although the relative importance of these cascades is still uncertain, it is clear that there are multiple ways to cause the modulation of gene expression that ultimately controls the gene response to the factor (ref. 31). These changes can be readily monitored by measuring changes in gene expression, both with immediate early response genes and secondary genes, that ultimately produce the characteristic physiological response (ref. 32). It is, after all, these differences in modulation of gene expression that determines the ultimate response of the cell to the original stimulus. In this regard, it is clear that there is a certain amount of redundancy in the various cascades. For example, it is possible to effectively eliminate PKC activation by diacylglycerol through pretreatment of the cells with phorbol esters (ref. 33). PC12 cells, a well known cultured cell line responsive to NGF, when treated in this fashion, will still produce neurites, the usual assay used to define responsiveness. This indicates that this cascade is not essential for the response to NGF in this particular cell line. In contrast, treatment of these same cells with othrovanadate, which blocks tyrosine dephosporylation 


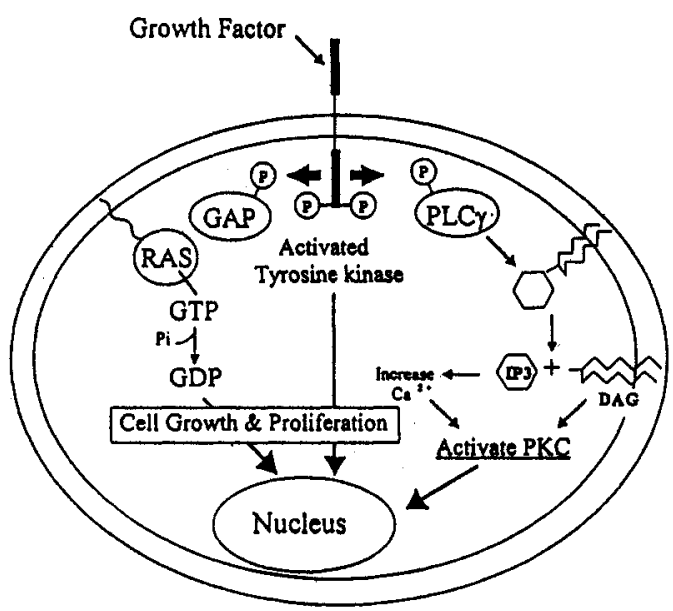

Fig. 1. Schematic representation of some general growth factor responses. Three pathways are indicated: 1)(left) the activation of RAS through the tyrosine phosphorylation (p) of G-protein activating protein (GAP); 2) (right) hydrolysis of phosphoinositides by the activation of $\mathrm{Cg}$ (PLCy) and 3)(middle) an unspecified pathway. Other abbreviations: IP3, inositol-1,4,5-triphosphate; DAG, diacylglycerol; PKC, protein kinase C.

through inhibition of the corresponding phosphates does block neurite proliferation, although apparently not through affecting the phosporylation induced by the receptor kinases. This suggests that non receptor kinases such as src which has been independently shown to be also required for the NGF response (ref. 27), are the targets of this compound and that dephosphorylation is as essential as phosphorylation in maintaining a viable response (ref. 34).

\section{TROPHIC VERSUS MITOGENIC RESPONSES}

One of the interesting questions in cell biology and in the understanding of the mechanism of action of polypeptide growth factors is the underlying differences between the signal transduction pathways that lead to trophic responses on the one hand and mitotic events on the other. Many growth factors can function in both capacities. For example, bFGF is a potent mitogen for virtually all cells which are derived from the mesoderm but can also act as an important neurotrophic factor for a variety of CNS neurons and PC12 cells (ref. 1,6). The FGF receptor family (Table 2) contains four basic types (as well as variations on the individual molecules), all of which contain tyrosine kinase entities. Furthermore, these receptors are manifested in both types of responsive cells. Thus, the phosphorylation events that can produce cell division on the one hand, or lead to neuron proliferation in the other cases, are dependent on the same potential activations.

As a means of approaching this central problem, we have investigated the response of PC12 cells to NGF and FGF, which induce neurite proliferation, and EGF, which is only mitotic for these cells. We have established that although the protein phosphorylation patterns are similar, there are clearly unique differences that are readily discernible in 1 and 2 dimensional gel analyses. Furthermore, we have identified a number of proteins, in cells labeled with ${ }^{35} \mathrm{~S}$-methionine, that are induced uniquely by NGF and FGF (in either positive or negative direction) but not by EGF. At the same time, a small number of proteins that are stimulated by all three growth factors have also been determined. These two groups of proteins represent potential candidates for components involved in the trophic response on the one hand and the mitogenic response on the other (all three proteins initially induce mitogenesis; only in the cases of NGF and FGF is the trophic response subsequently manifested)(H. Hondermarck, C.S. McLaughlin and R.A. Bradshaw, unpublished observations). The identification of these proteins is now in progress. While these may represent proteins involved in signal transduction process, they may also represent entities required for the manifestation of the physiological response and the differentiated (or mitotically induced) state. Similar studies are in progress for identifying the various differences in phosphorylation patterns. 


\section{PERSPECTIVES AND FUTURE DIRECTIONS}

There still remain many important areas of investigation before a complete description of the action of any polypeptide growth factor can be described. In particualr, the regulation of biosynthesis at both the transcriptional and post transcriptional levels, as well as a function in development, is poorly understood. This information will be essential in order to understand the role of growth factors in the context of the complete organism. At the same time, as already described, there remain many important functional enigmas that will require further resolution before we can appreciate the full mechanistic feature of the action of growth factors at the cellular level. Finally, there is an enormous potential for the use of growth factors themselves, as well as appropriate agonist and antagonists, as therapeutic agents in the tretament of a wide variety of pathologies. There have already been notable successes in this regard and one can anticipate, as further knowledge is obtained, that there will be increasing applications of growth factor technology to the practice of medicine.

\section{Acknowledgements}

The authors thank Gale Trudeau for help in the preparation of this manuscript. Portions of the work described emanating from the author's laboratory was supported in aprt by research grants from the US public Health Service (AG09735) and the American Cancer Society (BE-41). S.R was supported by a fellowship from the Bank of America-Giannini Foundation. Ralph A. Bradshaw thanks the Alexander Von Humboldt Foundation for support during the preparation of this manuscript.

\section{REFERENCES}

1. R. James and R. A. Bradshaw, Ann. Rev. Biochem. 53: 259-292 (1984).

2. R.A. Bradshaw and J.S. Rubin, J. Supramolecular Struct., 14: 183- 199, (1980).

3. Y. Yarden and A. Ullich, Ann. Rev. Biochem., 57: 443-478, (1988).

4. M. Sporn and A.B. Roberts (eds.), Polypeptide growth factors and their receptors, 1\&2, Springer -Verlag, Berlin, (1990).

5. R.A. Bradshaw, Trends Biochem. Sci., 14: 276-279, (1989).

6. P.A. Walicke, Ann. Rev. Neurosci., 12: 103-126, (1989).

7. R. Levi-Montalcini, Science 237: 1154-1164, (1987).

8. R.A. Bradshaw, Ann. Rev. Biochem. 47: 191-216, (1978).

9. R.H. Angeletti and R.A. Bradshaw, Proc. Natl. Acad. Sci. USA, 68: 2417-2420, (1971).

10. R.A. Bradshaw, T.L. Blundell, R. Lapatto, N.Q. McDonald and J. Murray-Rust, Trends Biochem. Sci., 18: 48-52, (1993).

11. R.H. Angeletti, R.A. Bradshaw, and R.D. Wade, Biochemistry, 10: 463-469, (1971).

12. N.Q. McDonald, R. Lapatto, J. Murray-Rust, J. Gunning, A. Wlodawer and T.L. Blundell, Nature, 354: 411-414, (1991).

13. C.F. Ibanez, T. Ebendal, G. Barbany, J. Murray-Rust, T.L. Blundell and H. Perrson, Cell, 69: 329-341, (1992).

14. M.B. Swindells, Science, 258: 1160-1161, (1992).

15. S. Daopin, G.H. Cohen, and D. Davies, Science , 258: 1161-1162, (1992).

16. S. Daopin, K.A. Piez, Y. Ogawa and D.R. Davies, Science, 257: 369-373, (1992).

17. M.P. Schlunegger and M.G. Grutter, Nature, 358 : 430-434, (1992).

18. C. Oefner, A.D'Arey, F.K. Winkler, B. Eggimann and M. Hosang, EMBO J., 11: 3921-3926, (1992).

19. J. Sclessinger and A. Ullrich, Neuron, 9: 1-20, (1992).

20. J. Massague, Cell, 69: 1067-1070, (1992).

21. L.R. Berkemeier, J.W. Winslow, D.R. Kaplan, K. Nikolics, D.V. Goeddel and A. Rosenthal, Neuron, 7: 857-866, (1991).

22. S.O. Meakin and E.M. Shooter, Trends Neurosci., 15: 323-331, (1992).

23. M.J. Radeke, T.P. Misko, C. Hsu, L.A. Herzenberg and E.M. Shooter, Nature 325: 593-597, (1987).

24. D. Johnson, A. Lanahan, C.R. Buck, A. Sehgal, C. Morgan, E. Mercer, M. Bothwell and M. Chao, Cell 47: 545-554, (1986).

25. M.V. Chao, Neuron, 9: 583-593, (1992).

26. A. Obermeier, H. Halfter, K.-H., Wiesmuller, G. Jung, J. Schlessinger and A. Ullrich, EMBO J., 12: 933-941, (1993).

27. S. Halegoua, R.C. Armstrong, and N.E. Kremer, Curr. Top. Microbiol. Immunol., 165: 119-170, (1991).

28. S. Raffioni, and R.A. Bradshaw, Proc. Natl. Acad. Sci USA, 89: 9121-9125, (1992).

29. M. Oshima, G. Sithanandam, U.R. Rapp and G. Guroff, J. Biol. Chem. 266: 23753-23760, (1991).

30. M. Ohmichi, L. Pang, S.J. Decker and A.R. Saltiel,J. Biol. Chem. 267: 1460414610, (1992).

31. S. Raffioni, S.E. Buxer and R.A. Bradshaw,Ann. Rev. Biochem., 62: 823-850, (1993).

32. J.G. Altin, D.A. Kujubu, S. Raffioni, D.D. Eveleth, H.R. Herschman and R.A. Bradshaw, J. Biol. Chem., 266: 5401-5406, (1991).

33. D. S. Reinhold and K. E. Neet, J. Biol. Chem. 264: 3538-3544, (1989).

34. Y. Y. Wu and R. A. Bradshaw, J. Cell Biol., 121: 409-422, (1993). 